\title{
Fixed-Target Experiments at HERA
}

\section{Measuring Spins with HERMES}

The deep-inelastic scattering of polarized electrons and muons from protons, deuterons and $3 \mathrm{He}$ has been used in several experiments in the past to study the origin of the spin of the nucleon. The spin-structure functions $g_{1}$ and $g_{2}$ can be determined experimentally by measuring the difference in cross-sections of polarized electrons on polarized nucleons between states where the spins are parallel and antiparallel. The measurement is possible because the polarized electron emits a virtual photon which interacts selectively with quarks and anti-quarks with a particular spin direction. The spin-structure functions are therefore experimentally derived from the asymmetry of the cross-section $\sigma$ for different relative spin orientations, $A^{\prime \prime}=\left(d \sigma^{\uparrow \downarrow}-d \sigma^{\uparrow \uparrow}\right) /\left(d \sigma^{\uparrow \downarrow}+d \sigma^{\uparrow \uparrow}\right)$. The Bjorken sum rule provides a relationship between the integral over the proton and neutron spin-structure functions and the weak-coupling constants, $g_{\mathrm{A}}$ and $g_{\mathrm{V}}$ (Ellis and Jaffe have also derived a similar sum rule). In the quark-parton model, the integral over the spin-structure function $g_{1}$ has a relatively simple interpretation:

$$
\begin{aligned}
& \int g_{1}^{p(n)}(x) \mathrm{d} x= \\
& \frac{1}{2}\left[\frac{4}{9} \Delta u(d)+\frac{1}{9} \Delta u(d)+\frac{1}{9} \Delta s\right]\left\{1-\alpha_{\mathrm{S}}\left(Q^{2}\right) / \pi\right\},
\end{aligned}
$$

where $\Delta u, \Delta d$ and $\Delta s$ represent the integrals over the quark momentum distributions of the up, down and strange quarks of the proton, $\Delta q=\int_{0}^{1}\left[q^{\dagger}(x)-\right.$ $\left.q^{\downarrow}(x)\right] \mathrm{d} x$. Here, $q^{\uparrow}(x)\left\{q^{\downarrow}(x)\right\}$ are the quark plus antiquark momentum distributions for the quark spin parallel \{anti-parallel\} to the spin of the nucleon.

The European Muon Collaboration has published a measurement of $\int_{0}^{1} g_{1}^{p}(x) \mathrm{d} x=0.126 \pm 0.010$ (stat.) \pm 0.015 (syst.) which is significantly smaller than the value predicted by Ellis and Jaffe of $0.175 \pm 0.018$. This result was interpreted as indicating that the contribution of the quarks to the proton spin is small, while the contribution from the sea of strange quarks is large. It has generated much experimental activity, from which first results have recently been published. The existing data do not contradict each other, within their large errors, but show that measurements at higher precision are needed.

A new, internal gas-target experiment HERMES to measure the spin-structure functions of proton and neutron has been approved for HERA. It will be able to determine the $x$-dependence of the polarized structure functions for $x$-values above 0.02 and $Q^{2}$ values between $1 \mathrm{GeV}^{2}$ and $12 \mathrm{GeV}^{2}$ with high precision; the detection of the leading hadrons will provide additional information. Furthermore, the experiment should provide access to other structure functions such as the transverse structure function $g_{2}(x)$ which provides information about the quark-gluon correlation. A test set-up of the experiment was mounted in early-1994 to study the influence of a gas target on the electron beam and the background conditions in the presence of the recently installed spin rotators used to polarize the beam (see page 93). Installation of the experiment and the start of data taking is foreseen for 1995.

\section{Exploring CP-Violation with HERA-B}

The violation of charge-parity symmetry has so far only been observed and studied in the kaon system. However, in spite of this work, the origin of the violation is not understood and it is therefore very important to study the phenomena on other systems. Neutral B-mesons provide such a system, and the analysis of certain decay modes will provide a crucial test of the underlying mechanism. In the framework of the Standard Model, the origin of CP-violation lies in a non-zero phase in the Cabibbo-KobayashiMaskawa matrix.

Most of today's knowledge about B-mesons comes from experiments at $\mathrm{e}^{+} e^{-}$colliders such as ARGUS (an experiment at DORIS which was ended last year) and CLEO. However, CP-violation cannot be explored at existing machines but requires a high-luminosity $\mathrm{e}^{+} \mathrm{e}^{-}$collider in which the energies of the two colliding beams are different. An "asymmetric B-factory" of this type will be built at SLAC in California, USA, and a second one at KEK in Tsukuba, Japan. Hadron accelerators provide an alternative source of Bmesons and a group centered around the DESY's ARGUS Collaboration has studied the possibility of measuring $\mathrm{CP}$-violation at HERA using a wire target in the halo of the HERA proton ring as a copious source of B-mesons.

Several decays of B-mesons can be used to study $\mathrm{CP}$-violation, with the decay $\mathrm{B}^{0} \rightarrow \mathrm{J} / \Psi \mathrm{K}_{\mathrm{s}}^{0}$ being especially suited, as its theoretical description has no uncertainties due to hadronic matrix elements. CP. violation leads in this decay to a time-dependent asymmetry in the decay rate of $\mathrm{B}^{0}$ and $\overline{\mathrm{B}} 0$ into $\mathrm{J} / \Psi \mathrm{K} \mathrm{K}_{\mathrm{S}}^{0}$,

$$
\begin{aligned}
A_{\mathrm{CP}} & =\frac{N\left(\mathrm{~B}^{0} \rightarrow \mathrm{J} / \Psi \mathrm{K}_{\mathrm{s}}^{0}\right)-N\left(\overline{\mathrm{B}}^{0} \rightarrow \mathrm{J} / \Psi \mathrm{K}_{\mathrm{s}}^{0}\right)}{N\left(\mathrm{~B}^{0} \rightarrow \mathrm{J} / \Psi \mathrm{K}_{\mathrm{s}}^{0}\right)+N\left(\overline{\mathrm{B}}^{0} \rightarrow \mathrm{J} / \Psi \mathrm{K}_{\mathrm{s}}^{0}\right)} \\
& =\sin (x t) \sin (2 \beta) .
\end{aligned}
$$

Here, $x=\Delta M / \Gamma=0.67$ (where $\Gamma$ is the decay width) characterizes the $\mathrm{B}^{0}, \overline{\mathrm{B}}^{0}$ flavour mixing and $t$ is the decay time measured in units of the $\mathrm{B}^{0}$-lifetime. The quantity $\sin (2 \beta)$ is a measure of the size of CP-violation and has an expected range of $0.16-0.98$. In order to perform a decisive measurement one has therefore to determine $\sin (2 \beta)$ with a precision of $\Delta \sin (2 \beta) \approx 0.05$.

The measurement of $A_{C P}$ requires a knowledge of the initial flavour of the decaying B-meson. Since the decay $\mathrm{B}^{0} \rightarrow \mathrm{J} / \Psi \mathrm{K}_{\mathrm{S}}^{0}$ leads to a CP-eigenstate, one cannot tell from the final state whether it originated from $a \mathrm{~B}^{0}$ or $\overline{\mathrm{B}}^{0}$. The initial flavour can be tagged however, either through the charge of leptons from the semileptonic decay of the second B in the event, or through the charge of a leading kaon from the decay of the second $\mathrm{B}$. In order to clearly identify the decay $\mathrm{B}^{0} \rightarrow \mathrm{J} / \Psi \mathrm{K}_{\mathrm{S}}^{0}$, only the decay of the $\mathrm{J} / \Psi$ into a pair of leptons is considered, as it provides a clear signature and can be used in a highly selective trigger.

The statistical error in the determination of the CPasymmetry $\sin (2 \beta)$ is given by $\Delta \sin (2 \beta) \approx(1 / D) \sqrt{K / N}$, where $N$ is the number of tagged events, $D$ the dilution factor originating from flavour mixing and wrong tagging, and $K$ a cut-dependent statistical factor arising from a cut in the decay time. To achieve a statistical error on $\sin (2 \beta)$ of 0.05 , about 3000 tagged and reconstructed events are needed. Taking into account the ratio of the b cross-section to the total inelastic cross-section, the probability to generate a $\mathrm{B}^{0}$ or $\overline{\mathrm{B}}^{0}$ in a b-event, the branching ratio for the decay $\mathrm{B}^{0} \rightarrow \mathrm{J} / \Psi \mathrm{K}_{\mathrm{S}}^{0}$, the leptonic $\mathrm{J} / \Psi$ branching ratio, the branching ratio for the decay $\mathrm{K}_{\mathrm{S}}^{0} \rightarrow \pi^{+} \pi^{-}$and estimates for efficiencies in triggering, tagging and reconstruction one finds that about $10^{15}$ interactions are needed to reach the required accuracy. Given the bunch-crossing rate of HERA of $10 \mathrm{MHz}$ and assuming 4 interactions per crossing, this corresponds to a data taking period of 5 years.

Studies made so far indicate that a significant measurement of $\sin (2 \beta)$ should be possible, and extensive experiments using a wire target in the halo of the HERA proton beam have already established that the required primary interaction rate can be obtained. The experiment, HERA-B, was recently approved provided certain conditions relating to funding and timely completion can be clarified by the end of 1994 .

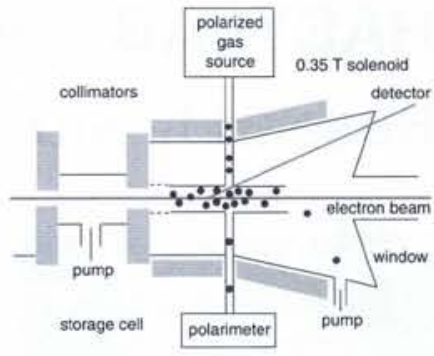

A schematic illustration of the HERMES internal gas-target experiment. It will use HERA's longitudinally polarized electron beam and a longitudinally polarized proton, deuteron or ${ }^{3} \mathrm{He}$ gas target. The target will be a long, windowless storage tube which is traversed by the beam and contains the polarized atoms in their pure atomic form, avoiding any dilution by other atoms. The plan is to polarize the hydrogen and deuterium atoms using an atomic beam method, while the ${ }^{3} \mathrm{He}$ atoms will be polarized using optical pumping.

An open spectrometer downstream of the target will measure the scattered electrons and the leading hadrons resulting from fragmentation of the target in coincidence with the scattered electron. Microstrip gas chambers and drift chambers in front of and behind the magnet, and proportional chambers inside the magnet, will monitor the trajectories of particles identified using a Cerenkov counter, a transition radiation detector and finally a lead-glass calorimeter.

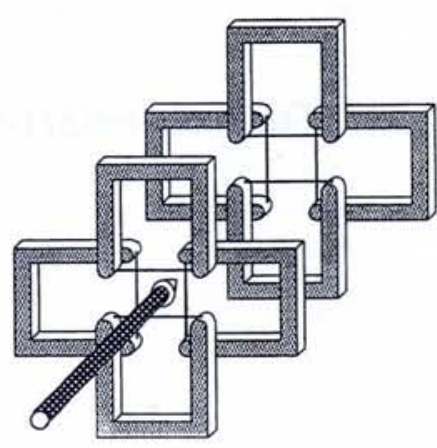

Layout of HERA-B's internal wiretarget experiment in the halo of HERA's proton beam. The requirements on a detector running under the conditions foreseen represent a major technical challenge. The detector has to be able to: reconstruct multiple events with as many as 50 tracks per bunch crossing at a $10 \mathrm{MHz}$ bunch crossing rate with high efficiency; reconstruct B-decay vertices; identify leptons and charged kaons; trigger rapidly and highly selectively on $\mathrm{J} / \Psi$. 